\title{
Human Red Blood Cells Microscope Morphology Analysis in High Glucose Condition
}

\author{
Bohua Feng ${ }^{a}$, Liufen Peng ${ }^{b}$ \\ College of Medical Information Engineering, Guangdong Pharmaceutical University, Guangzhou \\ 510006, China \\ atfengbh@163.com, bbandi-01@163.com
}

Keywords: Diabetic Nephropathy, Microscope Image Analysis, Morphological Characteristics.

\begin{abstract}
Microscope images analysis of red blood cells (RBCs) was well used for medical. Manual analysis of the images were subject to time-consuming and instability. Combined with computer image processing techniques in this research, a measuring and analysis system for microscope images(MIAS) of RBCs was developed. MIAS could recognize and measure RBCs shape parameters. Normal RBCs were compared with ones under high glucose. It indicated RBCs' areas, perimeters, major axis lengths, minor axis lengths, elongations, roundnesses and Feret diameters have difference between the two conditions. RBCs disk shapes changed into acanthocyte and stomatocyte. Fast and precise MIAS for RBCs contributed to pathogenesis of diabetic nephropathy(DN) research.
\end{abstract}

\section{Introduction}

The pathogenesis of diabetic nephropathy (DN) was complex. Blood rheology examination showed that the DN patients RBCs are in hypertonic condition due to high blood glucose levels. This enhanced the cell membrane protein glycosylation. And RBCs deformability, oxygen release amount and enzyme activity were definitely decreased.[1] These changes directly affected blood supply, resulting in renal tissue damage. Structure alterations in RBCs had important influence on their function and protein expression. Shape and structure parameters were contributed to understanding the pathogenesis, detection and control method of DN[2]. Computer image processing and pattern recognition technology of microscopic image had important significance in medical research. Compared to artificial way, computer image processing improved the efficiency, it enhanced the objectivity of the analysis[3]. In this paper, the MIAS achieved a variety methods of image processing, including image enhancement, point operations, color filling and edge detection. Searching center, counting, size and shape parameters calculating were carried out. Through a comparative study on results, abnormal RBCs parameters under high glucose condition were investigated.

\section{Experimental}

\subsection{Preparation of Phosphate Buffered Saline (PBS) and RBCs}

The 2.5g $\mathrm{NaH}_{2} \mathrm{PO}_{4} \cdot \mathrm{H}_{2} \mathrm{O}$, 22.49g $\mathrm{Na}_{2} \mathrm{HPO}_{4} \cdot 7 \mathrm{H}_{2} \mathrm{O}$ and $175.33 \mathrm{~g} \mathrm{NaCl}$ were dissolved in distilled water. The solution was moved into $1000 \mathrm{~mL}$ volumetric flask, adding distilled water to its full scale. The PBS solution was then placed in refrigerator under $4^{\circ} \mathrm{C}$. The $95 \mathrm{~mL}$ PBS was then blent with glucose $0.090 \mathrm{~g}$ and its $\mathrm{pH}$ was adjusted to 7.4. Amount of bovine serum albumin would be added to maintain RBCs shapes[4]. The venous bloods were collected from healthy adult (Group A, male, 34 years old), and DN patient (Group B, male, 58 years old, moderate and severe renal dysfunction with glomerular filtration rate (GFR) $15-60 \mathrm{ml} / \mathrm{min} \cdot 1.73 \mathrm{~m} 2$ )[5]. Samples anticoagulated with sodium heparin were removed white blood cells and platelets in upper layers after centrifugation. RBCs were then mixed with PBS (1:10). After centrifugalizing, young RBCs of Group A were obtained and suspended in PBS with glucose solution by the concentration of 20 $\mathrm{mmol} / \mathrm{L}$ and $5 \mathrm{mmol} / \mathrm{L}$. 


\subsection{Devices}

Nikon TE300 fluorescence phase contrast inverted microscope (40×, ph2), CCD camera (Samsung, Korea), thermostatic water bath, centrifuge, the sodium citrate anticoagulation $(0.109 \mathrm{~mol} / \mathrm{L}$, Sodium Citrate 1:9, Yangpu medical Co., Ltd.), transferpettors (Beijing Dragon lab), computer (Lenovo, Pentium Dualcore E5300 2.6GHz), Micrometer for microscope (scale division $0.01 \mathrm{~mm}$ ).

\subsection{RBCs Microscopic Image Processing and Characterization}

After the micrometer calibration, the RBCs slides were prepared and put on microscope stage in the constant temperature sample pool. The field diaphragm, cursory and accurate focusing were adjusted until a bright clear image was observed under $40 \times$ objective lens. Then the charge coupled device (CCD) was used to acquire the RBC images which would be converted into digital signal by image acquisition card. The digital signal was saved as computer image format file. Using the MIAS to measure the RBCs geometric parameters such as the contact area, perimeter, major axis length, minor axis length, elongation, roundness and Feret diameter, 15-20 RBCs were investigated at each glucose gradient concentration where repeated 3 times.

\section{Results and Discussion}

\subsection{Morphology and Structure of RBCs}

The RBC morphology is smooth edge discocyte of diameter 7-9 $\mu \mathrm{m}$, as shown in Fig. 1a and Fig. 2a.Under a certain glucose concentration, the RBCs shapes changed obviously. Though RBCs sizes were similar in Fig. 1a and b, the cells edges in Fig. 1b became irregular. Many RBCs were adhered to each other, some cells even showed stomatocyte shape, as shown in Fig. 1b. Further increasing the glucose concentration, a considerable number of RBCs size reduced and changed into the shape of acanthocyte [6] with lots of vesicles on cell membrane, as shown in Fig. 1c. The normal single living RBC shown in Fig. 2a was obviously more smooth and the acanthocyte one as shown in Fig. 2b. Therefore, glucose concentration had a significant effect on RBCs shape.

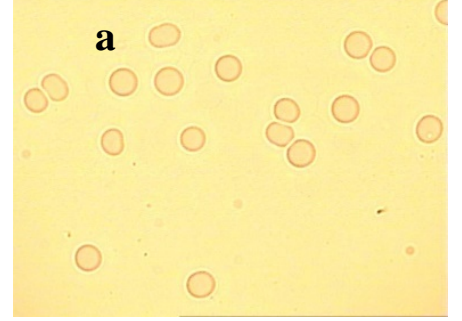

b

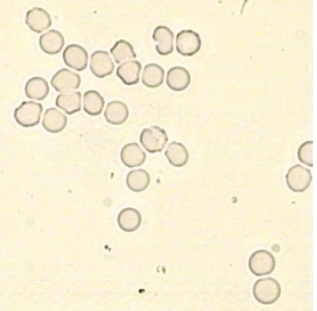

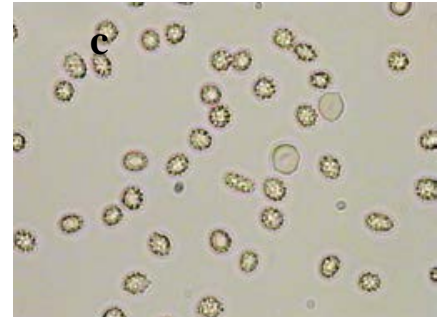

Fig. 1. Normal RBCs (a), RBCs under high glucose concentration (b), DN RBCs (c)
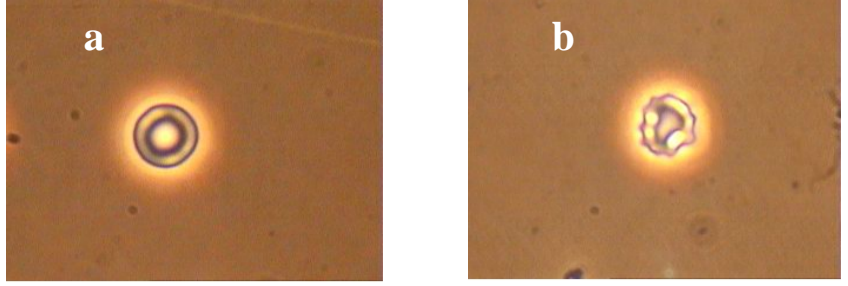

Fig. 2. A single living RBC in normal shape (a), under high glucose concentration (b)

\subsection{Microscope Images Enhancement and Binarization}

The RBCs images quality were usually low contrast and high noise, which had effect on image segmentation processing. Therefore, median filtering technique was applied to removing image noise. A pixel with its coordinates $(\mathrm{x}, \mathrm{y})$ was set as a center point in the microscope image, around which pixels distributed within the range of $3 \times 3$ area were sorted in ascending order of gray levels. And the mid-value of those pixels' gray levels was considered as the gray level of the center pixel. This procedure was achieved by transferring original image pixels' data to buffer, then affirmative mid-value of each pixel in buffer was copied to image to overwrite the original data. Thus, the RBCs in microscope images had more smooth edges and lower noise distinguished from background, which was contributed to further analysis. Image point operation was implemented by histogram thresholding method. The distribution of gray levels in microscope images were shown 
in Fig. 3a as a 2-dimension histogram, where horizontal ordinate referred to gray levels, and vertical coordinate represented gray levels frequency in RBCs images. The scope of gray levels threshold was easily adjusted in MIAS to achieve image binarization. And gray levels of points within the threshold range were marked by MIAS automatically. The closed regions referring to RBCs were filled by specified color as shown in Fig. 3a.

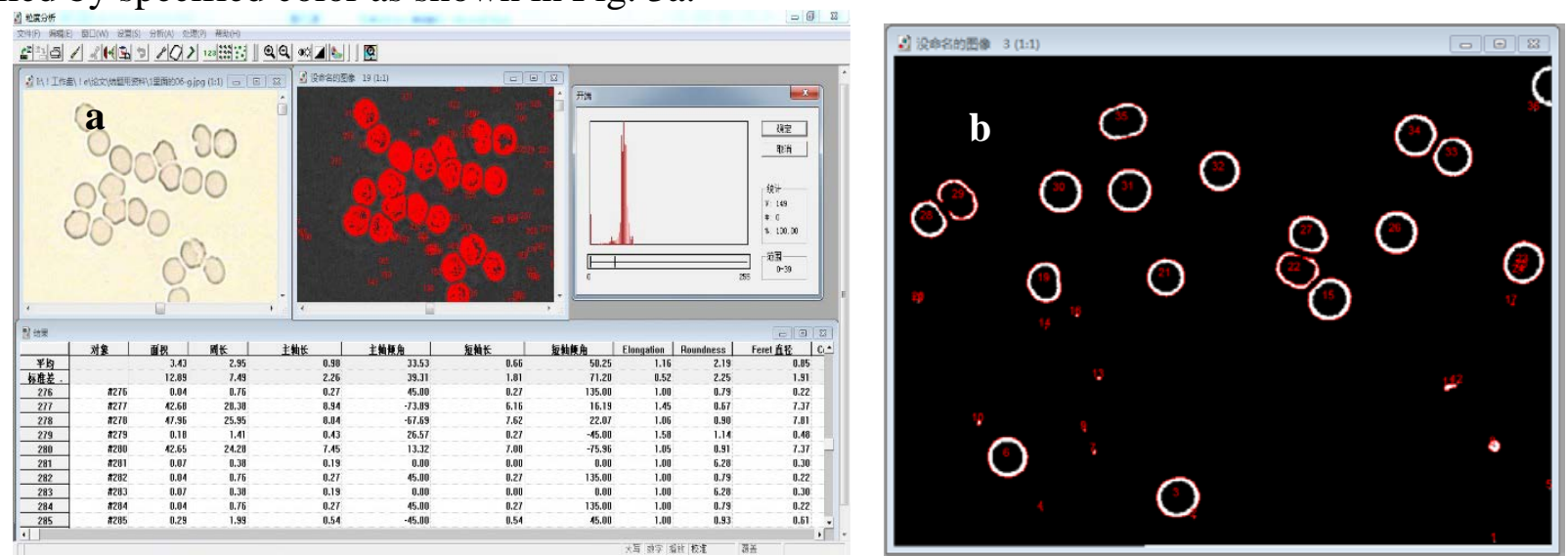

Fig. 3. Adjusting histogram thresholding and binarization (a), RBCs edges detection and marking

\subsection{RBCs Edges Detection Algorithm}

(b)

RBCs edges in microscope image were investigated by means of Sobel operator, which could decrease noise and obtain accurate edges[7]. Adjacent pixels gray level weighted difference reached the maximum value at RBCs edges. The operator contained two $3 \times 3$ convolution kernels, whose row and column number is odd. One was for detecting level edges, the other was for the vertical edges. After plane convolution with the operator, the image's horizontal and vertical brightness difference approximations were obtained. The calculation process was shown in scheme (1) to (3), where A represented original image, Gx and Gy separately standed for image edge detected in horizontal and vertical directions. Gray gradient value of single pixel was thus calculated in the two directions by scheme (2). Furthermore, gray gradient direction angle $\theta$ in scheme (3) was gained, where $\theta$ equaled to zero indicating a longitudinal edge of RBC with higher brightness in the right side of its edge.

$$
\begin{aligned}
& G_{x}=\left[\begin{array}{lll}
-1 & 0 & +1 \\
-2 & 0 & +2 \\
-1 & 0 & +1
\end{array}\right] * A \text { and } G_{y}=\left[\begin{array}{ccc}
+1 & +2 & +1 \\
0 & 0 & 0 \\
-1 & -2 & -1
\end{array}\right] * A \\
& G=\sqrt{G_{x}^{2}+G_{y}^{2}} \\
& \theta=\arctan \left(\frac{G_{y}}{G_{x}}\right)
\end{aligned}
$$

Some RBCs edges were blurred in the microscope image. As Sobel operator method was implemented, noise was eliminated and pixels of large gradient values were removed. Additionally, many small meaningless points should not be considered as RBCs. Corrosion and expansion functions were applied to remove these points in the binarized image (Fig. 3b). All those processes above prepared a clear image compared to the original one, and the RBCs were specifically recognized eliminating the noise. As shown in Fig. 3b, the RBCs were correctly selected by drawing a red line along their perimeters.

\subsection{RBC Shape Data Acquisition and Analysis}

Image analysis module aimed to search RBCs center point and calculate shapes parameters such as area, perimeter, major axis length, minor axis length, elongation, roundness and Feret diameter. After many times corrosions and thinning, image of obvious RBCs had been shown in Fig. 3, where the red color marked shapes made the center point searching and parameters calculation easier. The centers of RBCs were counted, shapes parameters were calculated using geometric formulas by MIAS. When RBCs were in normal glucose level solution $(\mathrm{pH}=7.4)$, the parameters were calculated and shown by MIAS in table1. And under the high glucose concentration, a stomatocyte shape cells parameters were listed in table 2 . 
Table 1 Normal RBCs shape parameters (unit: $\mu \mathrm{m}$ )

\begin{tabular}{llllllll}
\hline & Area $\left[\mu \mathrm{m}^{2}\right]$ & Perimeter & $\begin{array}{l}\text { Major Axis } \\
\text { Length }\end{array}$ & $\begin{array}{l}\text { Minor Axis } \\
\text { Length }\end{array}$ & Elongation & Roundness & $\begin{array}{l}\text { Feret } \\
\text { Diameter }\end{array}$ \\
\hline 1 & $48.84 \pm 3.09$ & $25.60 \pm 5.01$ & $8.14 \pm 0.30$ & $7.36 \pm 0.12$ & $1.11 \pm 0.06$ & $0.94 \pm 0.03$ & $7.89 \pm 0.08$ \\
2 & $45.40 \pm 4.71$ & $24.93 \pm 7.59$ & $7.65 \pm 0.15$ & $7.38 \pm 0.46$ & $1.04 \pm 0.06$ & $0.92 \pm 0$ & $7.60 \pm 0.23$ \\
3 & $50.74 \pm 6.00$ & $26.47 \pm 5.38$ & $8.30 \pm 0.07$ & $7.50 \pm 0.12$ & $1.11 \pm 0.07$ & $0.91 \pm 0.01$ & $8.04 \pm 0.40$ \\
4 & $39.62 \pm 6.84$ & $23.47 \pm 6.27$ & $7.63 \pm 0.07$ & $6.60 \pm 0.32$ & $1.16 \pm 0.05$ & $0.9 \pm 0.02$ & $7.10 \pm 0.20$ \\
5 & $14.15 \pm 6.16$ & $44.40 \pm 7.74$ & $8.17 \pm 0.50$ & $5.99 \pm 0.48$ & $1.36 \pm 0.05$ & $0.09 \pm 0.01$ & $4.24 \pm 0.21$ \\
6 & $47.92 \pm 3.48$ & $27.06 \pm 7.18$ & $8.18 \pm 0.24$ & $7.38 \pm 0.10$ & $1.11 \pm 0.01$ & $0.82 \pm 0.02$ & $7.81 \pm 0.42$ \\
7 & $50.70 \pm 5.11$ & $26.09 \pm 6.44$ & $8.12 \pm 0.36$ & $7.87 \pm 0.05$ & $1.03 \pm 0.08$ & $0.94 \pm 0.01$ & $8.03 \pm 0.04$ \\
8 & $35.57 \pm 6.72$ & $22.60 \pm 7.18$ & $7.12 \pm 0.47$ & $5.84 \pm 0.15$ & $1.22 \pm 0.04$ & $0.87 \pm 0.01$ & $6.73 \pm 0.03$ \\
9 & $12.83 \pm 6.88$ & $43.35 \pm 5.91$ & $8.40 \pm 0.05$ & $6.10 \pm 0.42$ & $1.38 \pm 0.05$ & $0.09 \pm 0.02$ & $4.04 \pm 0.09$ \\
10 & $46.24 \pm 4.68$ & $25.39 \pm 6.91$ & $7.71 \pm 0.17$ & $7.45 \pm 0.36$ & $1.03 \pm 0.06$ & $0.90 \pm 0.05$ & $7.67 \pm 0.14$ \\
11 & $42.15 \pm 5.81$ & $24.52 \pm 5.36$ & $7.39 \pm 0.26$ & $7.21 \pm 0.36$ & $1.02 \pm 0.04$ & $0.88 \pm 0.04$ & $7.33 \pm 0.31$ \\
12 & $49.31 \pm 3.42$ & $27.08 \pm 6.16$ & $8.03 \pm 0.48$ & $7.76 \pm 0.20$ & $1.03 \pm 0.08$ & $0.84 \pm 0.02$ & $7.92 \pm 0.45$ \\
Mean & 42.08 & 27.53 & 7.95 & 7.08 & 1.13 & 0.8 & 7.22 \\
St. Dev & 11.79 & 6.32 & 0.5 & 0.7 & 0.14 & 0.27 & 1.23 \\
\hline
\end{tabular}

Table 2 RBCs shape parameters under high glucose concentration (unit: $\mu \mathrm{m}$ )

\begin{tabular}{llllllll}
\hline & Area $\left(\mu \mathrm{m}^{2}\right)$ & Perimeter & $\begin{array}{l}\text { Major Axis } \\
\text { Length }\end{array}$ & $\begin{array}{l}\text { Minor Axis } \\
\text { Length }\end{array}$ & Elongation & Roundness & $\begin{array}{l}\text { Feret } \\
\text { Diameter }\end{array}$ \\
\hline 1 & $51.54 \pm 4.44$ & $27.16 \pm 7.32$ & $8.24 \pm 0.01$ & $8.06 \pm 0.31$ & $1.02 \pm 0.07$ & $0.88 \pm 0.04$ & $8.10 \pm 0.41$ \\
2 & $49.68 \pm 3.04$ & $27.96 \pm 7.46$ & $8.76 \pm 0.06$ & $7.80 \pm 0.36$ & $1.12 \pm 0.06$ & $0.80 \pm 0.04$ & $7.95 \pm 0.30$ \\
3 & $47.23 \pm 4.24$ & $25.65 \pm 6.84$ & $7.99 \pm 0.34$ & $7.72 \pm 0.45$ & $1.03 \pm 0.09$ & $0.90 \pm 0.03$ & $7.75 \pm 0.39$ \\
4 & $45.29 \pm 4.95$ & $25.01 \pm 6.02$ & $8.34 \pm 0.03$ & $7.11 \pm 0.24$ & $1.17 \pm 0.03$ & $0.91 \pm 0.05$ & $7.59 \pm 0.11$ \\
5 & $43.79 \pm 7.51$ & $26.76 \pm 6.16$ & $8.14 \pm 0.46$ & $6.98 \pm 0.02$ & $1.17 \pm 0.04$ & $0.77 \pm 0.04$ & $7.47 \pm 0.33$ \\
6 & $42.26 \pm 5.33$ & $24.02 \pm 5.94$ & $7.92 \pm 0.09$ & $6.57 \pm 0.49$ & $1.21 \pm 0.01$ & $0.92 \pm 0.03$ & $7.34 \pm 0.16$ \\
7 & $41.31 \pm 6.87$ & $24.40 \pm 7.69$ & $7.39 \pm 0.01$ & $7.20 \pm 0.39$ & $1.03 \pm 0.02$ & $0.87 \pm 0.03$ & $7.25 \pm 0.32$ \\
8 & $40.72 \pm 5.42$ & $24.52 \pm 5.35$ & $7.74 \pm 0.37$ & $6.96 \pm 0.27$ & $1.11 \pm 0.05$ & $0.85 \pm 0.01$ & $7.20 \pm 0.12$ \\
9 & $36.96 \pm 4.74$ & $23.43 \pm 6.55$ & $7.58 \pm 0.46$ & $6.63 \pm 0.06$ & $1.14 \pm 0.01$ & $0.85 \pm 0.04$ & $6.86 \pm 0.25$ \\
10 & $23.61 \pm 3.45$ & $51.54 \pm 6.37$ & $8.86 \pm 0.08$ & $6.12 \pm 0.17$ & $1.45 \pm 0.03$ & $0.11 \pm 0.01$ & $5.48 \pm 0.11$ \\
11 & $17.88 \pm 6.76$ & $42.58 \pm 5.09$ & $7.58 \pm 0.26$ & $6.22 \pm 0.31$ & $1.22 \pm 0.06$ & $0.12 \pm 0.01$ & $4.77 \pm 0.32$ \\
12 & $14.29 \pm 6.79$ & $35.42 \pm 7.35$ & $6.89 \pm 0.11$ & $6.74 \pm 0.23$ & $1.02 \pm 0.05$ & $0.14 \pm 0.04$ & $4.27 \pm 0.01$ \\
Mean & 36.34 & 32.06 & 7.95 & 7.09 & 1.12 & 0.65 & 6.72 \\
St. Dev & 10.62 & 14.41 & 1.27 & 0.52 & 0.14 & 0.35 & 1.1 \\
\hline
\end{tabular}

Under high glucose concentration, many RBCs adhered to each other, cells shapes changed to stomatocyte, the roundness parameters (average value 0.65) were lower than that of low concentration. Roundness average value in table 1 reached 0.8 that implied the shapes are closed to discocyte. Feret diameter parameters also showed that RBCs became smaller under high glucose environment (Fig. 1b, c). Average Feret diameter in table 1 was $7.22 \mu \mathrm{m}$ that standed for a normal RBC size. This parameter was closed to the values of major axis length and minor axis length listed in the same table, which also suggested that the shape of RBCs in high glucose environment has less regularity than the normal ones. Areas were around $42 \mu \mathrm{m}^{2}$ that was more homogeneous than the values in table 2 . Accordingly, a few of smaller areas values were observed in table 2, the data were thus more volatile. And this was consistent with the Fig.1b and c, where many stomatocyte and adherion RBCs were found. There were no obviously changes in average values of major axis length and minor axis length in both table, but the elongation values were different significantly. A number of elongation values were bigger up to 1.59 in Fig. 2. It suggested that higher glucose concentration may reduced $\mathrm{pH}$, RBCs shapes in such acidulated solution would turn to stomatocyte and acanthocyte (Fig. 1b and c). Therefore, changes of glucose concentrations had obviously effect on RBCs shapes.

\section{Summary}

The MIAS for RBCs was used to measure the morphological parameters of RBCs under normal and high glucose. Comparison of the results showed that RBCs microscopic image analysis technology was efficient on processing cells edge recognition, calculating important cells morphological parameters. Those accurate quantitative results well reflected the morphologic changes of RBCs during certain glucose concentrations. Under different glucose concentrations, the changes of the 
morphological parameters of RBCs reflected altered membrane protein and lipid bilayer structures. Accordingly, the stability and deformation of the RBCs membranes and the performance of RBCs gases transport were changed. Furthermore, a series of diabetic complications, for example DN, may have close related with those changes. With the advantages of computer image processing, fast and accurate measuring and processing images were realized while the inconvenience of manual way was avoided. And for predicting the clinical course of DN even evaluating follow-up effect of drug treatment, the MIAS had provide a quick, intuitive and accurate approach.

\section{Acknowledgments}

This project is supported by Medical Scientific Research Foundation of Guangdong Province (B2014203) and Science\&Technology Planning Project of Guangdong Province, China (2016A020215162).

\section{References}

[1] P. D. Stein, J. Goldman, F. Matta and A. Y. Yaekoub, Diabetes Mellitus and Risk of Venous Thromboembolism, American Journal of the Medical Sciences. 337 (2009) 259-264.

[2] J. G. Yong, P. Ruan, B. H. Feng, M. L. Shi and L. F. Peng, Study on the relevance of changes in red blood cell morphology and function of patients with diabetic nephropathy to the development of diabetes, Journal of Jinan University (Natural Science \& Medicine Edition). 34 (2013) 610-614.

[3] J. Jiang, D. N. Fan, W. Chen and Z. H. Xiong, Erythrocyte counting system based on blood micrograph, Medical Information. 22 (2009) 8-10.

[4] C. C. Yao, Y. X. Huang, X. K. LI and P. Ruan, Effects of $\mathrm{pH}$ on structure and function of single living erythrocyte, Chinese Science Bulletin. 48 (2003) 1342-1346.

[5] N. K. Foundation, K/DOQI clinical practice guidelines for chronic kidney disease: evaluation, classification, and stratification, Annals of Internal Medicine. 139 (2003) 137-147.

[6] J. C. Wang and Z. W. Shi, The significance of urine red blood cell morphology analysis in the differential diagnosis on diabetic nephropathy and non diabetic nephropathy, Journal of Practical Medicine. 31 (2015) 95-97.

[7] H. Li, Edge detection technology of image processing, Agriculture \& Technology. 30 (2010) 163-165. 